\title{
INSTABILITY AT THE PAL LOCUS IN ANTIRRHINUM MAJUS
}

\section{A GENE CONTROLLING MUTATION FREQUENCY}

\author{
B. J. HARRISON * and J. R. S. FINCHAM† \\ The John Innes Institute, Bayfordbury, Hertford, Herts
}

Received 18.iv.67

\section{INTRODUGTION}

THE unstable allele pallida-recurrens ( pal $^{r e c}$ ), in its unmutated and homozygous state, determines an absence of anthocyanin pigment. It mutates to a range of other pal alleles which differ from each other in stability and in pigmentforming capacity. Different palrec stocks differ very greatly in mutation frequency, as expressed both in pigmented spots and flakes on the flower and in germinal mutations resulting in whole-mutant seedlings. Two palrec stocks in our collection, called by us (low) pal rec and (high) palrec, differ in mutation frequency by a factor of more than 100 . In a previous paper (Harrison and Fincham, 1964) it was shown that the difference between these stocks behaved in crosses as if due to one major gene, but the location of this gene was not determined. In this paper the earlier results are confirmed, and it is shown that the gene controlling mutation frequency is unlinked to pal.

Three basic stocks were used in this study. (Low) palrec and (high) palrec have been referred to above. A third stock, originally supplied to us by Dr D. R. Sampson of the Canadian Department of Agriculture, is homozygous for pal-tubocolorata $\left(p^{\text {tub }}\right.$ ), a recessive stable allele giving flowers which are completely acyanic except for a ring of pigment at the base of the corolla beneath the calyx. It was shown previously (Harrison and Fincham, 1964) that the genetic background of the paltub stock was particularly favourable to high mutation frequency of palrec. All three stocks have been maintained by self-pollination for many generations.

\section{GROWTH OF PLANTS}

Family numbers are preceded by letters indicating the year and season in which they were grown. $\mathrm{R}$ and $\mathrm{V}$ families were grown in an outside plot, flowering in June to August. $\mathrm{T}$ and $\mathrm{W}$ families were grown in winter and early spring in the greenhouse, sometimes with the help of mercury vapour lights.

\section{Methods OF SCORING SPOT FREQUENGY}

Plants were scored subjectively as " high ", "medium" and "low". In families resulting from back-crosses to " high " the segregation of " high" and "medium", where it occurred, was very clear and unambiguous. In families resulting from selfing, and segregating " high", " medium" and "low", classification was more difficult and, in borderline cases, was occasionally somewhat arbitrary. Subjective assessment of spot frequency

* Present address: The John Innes Institute, Colney, Norwich.

† Present address: Department of Genetics, The University, Leeds, 2. 
was often checked by actual spot counts. The inner surface of one of the two upper corolla lobes of a representative flower was counted for each plant. When the spot number was reasonably low this area was counted completely, but when the number was too high for exact counting a known fraction of the total area was counted (in a lane of defined width running across the lobe) and the resulting spot count was multiplied by the appropriate factor. Representative counts are cited in several of the tables to give an idea of the degree of difference between the various classes. It is not possible to define "high", "low" and "medium" in absolute terms, since mutation frequency in all pal rec genotypes depends critically on temperature (Harrison and Fincham, 1964). However, table 3 shows the range of spot numbers observed in the different categories under one set of conditions.

\section{Results}

(i) Effect of a Stabiliser gene on the palrec allele from original low-spotted stock

The present investigation was started by observations on four families. R73 was obtained by selfing a palrec/pal ${ }^{t u b}$ plant. This plant had a somewhat complicated pedigree, but its genetic background was substantially derived from the pal ${ }^{t u b}$ stock; it showed a comparatively low spot frequency

TABLE 1

The families in which segregation of $\mathrm{St} / \mathrm{st}$ was first recognised

\begin{tabular}{|c|c|c|c|c|c|}
\hline \multirow[b]{2}{*}{$\begin{array}{c}\text { Family } \\
\text { No. }\end{array}$} & \multirow[b]{2}{*}{ Parentage } & \multicolumn{4}{|c|}{ Numbers in each class $\uparrow$} \\
\hline & & $\begin{array}{c}\text { High } \\
(2500+)\end{array}$ & $\begin{array}{r}\text { Medium } \\
(100-500)\end{array}$ & $\begin{array}{c}\text { Low } \\
(c a .20)\end{array}$ & $\begin{array}{c}\text { Acyanic } \\
p^{t} l^{t u b} / p a l^{t u b}\end{array}$ \\
\hline R73 & palrec/paltub St/st* selfed & 8 & 13 & 6 & 7 \\
\hline R79 & pal rec $^{\prime} / p a l^{t u b} S t / s t^{*} \times p a l^{t u b}$ stock $(s t / s t)$ & 7 & 17 & 0 & 16 \\
\hline $\mathbf{R} 80$ & 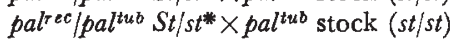 & 7 & 10 & 0 & 20 \\
\hline $\mathbf{R} 84$ & $\mathrm{pal}^{r \mathrm{sc}} / \mathrm{pal} \mathrm{l}^{\mathrm{tub}} \mathrm{St} / \mathrm{st} * \times p a l^{t u b}$ stock $(\mathrm{st} / \mathrm{st})$ & 11 & 9 & 0 & 18 \\
\hline
\end{tabular}

presumably inherited from (low) pal ${ }^{r e c}$, from which stock the pal ${ }^{r e c}$ allele had been derived. R73 segregated 25 spotted plants to 7 homozygous pal ${ }^{t u b}$ and, among the spotted plants, there were 8 with high, 13 with medium and 6 with low spot frequency.

R79, R80 and R84 were obtained by crossing medium-spotted palrec/ pal ${ }^{t u b}$ plants to the pal ${ }^{t u b}$ stock. The heterozygous parents of R79 and R80 were from a uniform medium-spotted family obtained by crossing to pal ${ }^{t u b}$ a low-spotted plant from the $\mathrm{F}_{2}$ of (low) palrec $\times$ paltub. The heterozygous parent of R84 was from a family, heterogeneous with respect to spot frequency, obtained by crossing to pal ${ }^{t u b}$ a medium-spotted plant from the same $F_{2}$. R79, R80 and R84 all showed a clear segregation of high and low to medium-spotted plants (table 1 ).

These initial observations strongly suggested the existence of a semidominant anti-mutagenic or stabilising gene, which we will call St, unlinked to pal. The paltub stock was apparently homozygous for the non-stabilising allele st, and the parents of R73 as well as the heterozygous parents of R79, R80 and R84 all appeared to be heterozygous St/st as well as palrec/pal ${ }^{t u b}$. The remaining crosses reported below were designed to test this hypothesis and further to assess the effect of the $S t / s t$ allelic difference. 
Medium-spotted plants from families R79 and R80 were back-crossed to the pal ${ }^{t u b}$ stock, and the resulting families (T178-181 and T185-187) all segregated acyanic (homozygous pal ${ }^{\text {tub }}$ ), high-spotted and medium-spotted plants (table 2). The approach to the expected 2:1:1 ratio of acyanic highspotted and medium-spotted plants was not close (136:100:64 in aggregate). There was a deficiency of both acyanic and medium-spotted plants. However, the clear segregation of the two spotted classes (plate I) supported the

TABle 2

$R 79$ and R80 plants backcrossed to paltub stock

\begin{tabular}{|c|c|c|c|c|}
\hline \multirow[b]{2}{*}{$\begin{array}{c}\text { Family } \\
\text { No. }\end{array}$} & \multirow[b]{2}{*}{ Plant tested } & \multicolumn{3}{|c|}{ Numbers in each class } \\
\hline & & High & Medium & Acyanic $p a l^{t u b} / p a l^{t u b}$ \\
\hline T178 & $\mathrm{R} 79^{3}$ (medium) & 33 & 21 & 48 \\
\hline T179/80 & R795 (medium) & 34 & 21 & 43 \\
\hline T181 & R797 (medium) & $3 \dot{s}$ & 22 & 45 \\
\hline $\mathrm{T} 182 / 3$ & R7910 (high) & $35^{*}$ & 0 & 33 \\
\hline T184 & R80 19 (high) & 35 & 0 & 32 \\
\hline
\end{tabular}

* Plus 10 full-red mutants.

hypothesis of a single major controller gene $(S t)$ and encouraged the belief that the deficiency in the medium-spotted class was due to differential viability or mutation, or both. It was clear that pal and $S t$ were unlinked since the recombinant high-spotted class outnumbered the parental-type medium-spotted class. High-spotted plants, similarly back-crossed, gave good approximations to $1: 1$ ratios of high-spotted to acyanic, with no low or medium-spotted progeny (table 2). In addition, a very low-spotted R73 plant of presumptive genotype pal ${ }^{r e c} / \mathrm{pal}^{\mathrm{tub}} \mathrm{St} / \mathrm{st}$ was selfed to give family V22. As expected, only low-spotted plants were produced in this family (table 3 ).

As a further check, and in order to observe segregation of $S t / S t$ and $s t / s t$ plants in the same family, several medium-spotted plants from the $\mathrm{T}$ families were selfed. The scoring of the resulting families is shown in the lower part of table 4 . While the classification wascomplicated by the presence of both homozygous and heterozygous palrec in each class, and it is quite likely that some plants were misclassified, there was a reasonable segregation of low-, medium- and high-spotted plants, though there were too many " highspotted "plants for a good fit to a $1: 2: 1$ ratio. Whether this deviation from the expected result was due to misclassification of some $S t / s t$ plants as " high " instead of "medium", to mutation of $S t$ to $s t$ (see below) or to viability disturbance is not known.

(ii) Effect of $\mathrm{St}$ on the $\mathrm{pal}^{\mathrm{rec}}$ allele from the original high-spotted stock

As has been mentioned previously (Harrison and Fincham, 1964), the $\mathrm{F}_{2}$ generation from the cross between the standard (high) palrec and pal tub stocks contained plants which were more densely spotted than the original (high) palrec stock itself. This is regarded as indicating that (high) palrec carries a gene or genes having some depressing effect on mutation frequency, though not nearly so marked an effect as that of $S t$ in the (low) pal rec stock. The most densely spotted $\mathrm{F}_{2}$ plants breed true on selfing, apart from producing a very high frequency of pal mutants of various kinds. Very densely spotted lines so established are referred to collectively as (very high) palrec and will 
not be otherwise distinguished here. Plants of these lines were used as testers for the presence of $S t$ in plants homozygous for pal ${ }^{t u b}$.

Five paltub/paltub plants from family R73 were crossed to (very high) palrec, and the results are shown in table 3. It is evident that one of the 5 pal ${ }^{t u b}$ plants (that which gave family V30) must have been st/st, one (which gave family T171) St/St and the remaining three $S t / s t$. Several of the mediumspotted T171 plants (presumed constitution palrec/paltub St/st) were selfed, and the scoring of the resulting families (W64-67) is summarised in the upper

TABLE 3

$R 73$ plants selfed or crossed to $\mathrm{palrec} / \mathrm{palrec}$ st/st testers

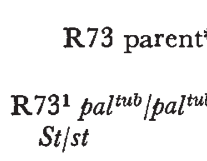

R73 ${ }^{13}$ paltub $^{t \text { paltub }}$ $(S t / S t)$

\section{R7320 ${ }^{20 l^{t u b}} / \mathrm{pal}^{\mathrm{tub}}$} $(S t / s t)$
R73 ${ }^{24} p a l^{t u b} \mid p a l^{t u b}$ (St/st)

$\mathrm{R} 73^{30} \mathrm{pal}^{\mathrm{tub}} / \mathrm{pal}^{\mathrm{tub}}$ st $/$ st

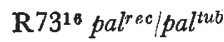
St/St selfed
Numbers in each class

\begin{tabular}{|c|c|c|c|c|c|}
\hline \multirow{2}{*}{$\begin{array}{c}\text { Family } \\
\text { No. } \dagger\end{array}$} & \multicolumn{5}{|c|}{ Numbers in each class } \\
\hline & High & Medium & Low & Mutant§ & Acyanic \\
\hline $\mathrm{T} 170$ & 7 & 9 & - & 10 & - \\
\hline V29 & $\begin{array}{c}21(1540-) \\
(6700)\end{array}$ & $\begin{array}{c}12(140-) \\
(270)\end{array}$ & 一 & 0 & - \\
\hline $\mathrm{T} 171$ & 0 & 23 & - & 30 & - \\
\hline V31 & $\begin{array}{c}29(4400-) \\
(8900)\end{array}$ & $\begin{array}{c}23(260-) \\
(760)\end{array}$ & - & 0 & - \\
\hline T172/3 & 18 & 39 & - & 4 & - \\
\hline V34 & $\begin{array}{c}15(900-) \\
(2800)\end{array}$ & $\begin{array}{c}14(77-) \\
(180)\end{array}$ & - & 0 & - \\
\hline V30 & $\begin{array}{c}32(13,000-) \\
(7000)\end{array}$ & $\begin{array}{c}0(77-) \\
(180)\end{array}$ & - & 0 & - \\
\hline
\end{tabular}

12 palrec/palrec (4-21)

12 pal $^{\text {tub }} /$ pal rec $(2-19)$

* Crossed to (very high) palrec tester except where indicated.

$\dagger \mathrm{T}$ families were grown during the winter and spring in the greenhouse; $\mathrm{V}$ families during spring and summer outdoors. $\mathrm{V}$ and $\mathrm{T}$ families were from different capsules when from the same cross.

$\$$ Numbers in parenthesis are the range of spot numbers found on counting representatives flowers from all (or nearly all) plants of the family.

$\S$ Mutants (mostly full reds) from mutation in the $p a l^{r e c} / p a l^{r e c}$ st/st parent.

part of table 4. The results were very similar to those obtained from plants of similar constitution but with the pal ${ }^{r e c}$ allele derived originally from the (low) palrec stock (lower part of table 4). Plate II shows a comparison of $S t / S t, S t / s t$ and $s t / s t$ plants with their palrec alleles derived from the two different sources. It is evident that any difference at the pal locus itself has a very small effect compared with that of the $S t / s t$ difference.

\section{(iii) Quantitative assessment of the St-st difference}

st/st pal ${ }^{r e c}$ and $S t / s t$ pal ${ }^{r e c}$ plants were usually clearly distinct in families in which the two genotypes were segregating, but $S t / s t$ and $S t / S t$ plants were less so. While $S t$ is by no means completely dominant, the heterozygote is closer to the $S t / S t$ than to the $s t / s t$ homozygote. Ranges of spot frequency, based on flowers selected as being representative of the various subjectively defined classes are shown in tables 1 and 3. The most complete counts were 
made on the $\mathrm{V}$ families which were grown outdoors in adjacent plots and which flowered at almost the same time. It will be seen that there was virtually no overlap between the different $S t$ genotypes in these families, and that, very roughly speaking, the spot frequencies of palrec/pal ${ }^{\text {tub }}$ plants in the $S t / S t$, st/St and st/st classes averaged 10, 200 and 4000 respectively. However, there was as much as a 2- to 3-fold variation within each class.

TABLE 4

Results of selfing $\mathrm{palrec}^{\mathrm{re}} \mathrm{paltub} \mathrm{St} / \mathrm{st}$ plants

Numbers in each class*

\begin{tabular}{|c|c|c|c|c|c|c|}
\hline $\begin{array}{c}\text { Family } \\
\text { No. }\end{array}$ & $\begin{array}{l}\text { Parent } \\
\text { plant }\end{array}$ & High & $\begin{array}{c}\text { Medium } \\
(100-1000)\end{array}$ & $\begin{array}{c}\text { Low } \\
(0-20)\end{array}$ & $\begin{array}{c}\text { Total } \\
\text { spotted }\end{array}$ & $\underset{\left(p a l^{t u b} / p a l^{t u b}\right)}{\text { Acyanic }}$ \\
\hline W64 & $\mathrm{T} 171^{1}$ & 21 & 19 & 28 & 68 & 16 \\
\hline W65 & $\mathrm{T} 171^{10}$ & 18 & 31 & 25 & 74 & 22 \\
\hline W66 & $\mathrm{T} 171^{12}$ & 15 & 41 & $19 \dagger$ & 75 & 21 \\
\hline \multirow[t]{2}{*}{ W67 } & $\mathrm{T} 171^{16}$ & 0 & 8 & 1 & 9 & 6 \\
\hline & Totals & 54 & 99 & 73 & 226 & 65 \\
\hline W68 & TI784 & 3 & 24 & 13 & 40 & 12 \\
\hline W69 & $\mathrm{T} 178^{8}$ & 26 & 23 & 17 & 66 & 21 \\
\hline W70 & T178 & 5 & 8 & 3 & 16 & 3 \\
\hline W73 & Т1 $79^{22}$ & 12 & 26 & 9 & 47 & 24 \\
\hline W78 & $\mathrm{T} 180^{2}$ & 25 & 34 & 17 & 76 & 17 \\
\hline W79 & $T 181^{4}$ & 36 & 29 & 10 & 75 & 21 \\
\hline W80 & $\mathrm{T} 181^{43}$ & 29 & 35 & 13 & 77 & 18 \\
\hline \multirow[t]{2}{*}{ W81 } & $\mathrm{T} 181^{58}$ & 16 & 35 & 8 & 59 & 20 \\
\hline & Totals & 152 & 214 & 90 & 456 & 136 \\
\hline
\end{tabular}

* Numbers in parenthesis show the range of spot count found in flowers selected as representative of each class.

$\dagger$ Includes 2 fully coloured mutants.

† All medium-spotted.

In the $s t / S t$ and $S t / S t$ genotypes, where the proportion of mutant cells in the flowers was small, it was expected that palrec/palrec plants should, on average, have twice as many spots as palrec/paltub plants. Our observations are not inconsistent with this but sufficient counts on pal ${ }^{r e c} / p a l^{r e c} S t / s t$ flowers have not been made to establish the point. Spot frequencies in $S t / S t$ flowers are very low and seem to be rather variable whether palrec is homozygous or heterozygous. Plants of constitution $\mathrm{pal}^{\mathrm{rec}} / \mathrm{pal} \mathrm{l}^{\mathrm{rec}} \mathrm{st} / \mathrm{st}$ are normally very densely spotted indeed and it is doubtful whether meaningful spot counts can be made.

\section{(iv) Possible mutation of $\mathrm{St}$}

Occasional sectors showing the st/st palrec phenotype are found on $S t / s t$ palrec plants (plate III). This could be due either to mutation of palrec to a state unresponsive to $S t$ or, perhaps more plausibly, to mutation of $S t$ to a state resembling st. Such mutation could be a factor contributing to the excess of $s t / s t$ over $S t / s t$ plants observed in some families in which a $1: 1$ ratio was expected (table 2 ).

\section{Discussion}

The stabiliser gene demonstrated in this paper could be compared with the normal allele $d t$ of $D$ otted $(D t)$ investigated by Rhoades (1941) in maize. 
The dominance relationships in the two cases are not dissimilar; st/st produced many more than twice as many mutations as does $S t / s t$, while in the Dotted case, where one, two or three doses of $D t$ can be obtained in the triploid endosperm, two doses give about 3 times, and three doses about 30 times, as many spots as one dose. The main difference is that, in the absence of $D t, a_{1}$ does not mutate with observable frequency, while palrec does mutate, though at a comparatively low rate, with homozygous Stab. Whether one attributes a stabilising action to one allele or a mutating function to the other is a mere matter of semantics, so long as we do not understand the mechanisms involved. One can also compare st with the Modifier gene in maize described by McClintock (1965) which enhances the mutation frequency of $a_{1}^{m-1}$ in the presence of Suppressor-mutator $(S p m)$. This is in one way a closer parallel, since McClintock's Modifier, like $s t$, merely enhances. mutation frequency, rather than inducing mutations where none would occur without it. On the other hand, Modifier appears to be fully dominant, unlike st which is nearer recessive than dominant. Modifier, like other controlling elements in maize, undergoes transpositions; this phenomenon has not been looked for in our material.

A question of obvious interest concerns the specificity of the $S t / s t$ system. So far it has been shown to act only on palrec. It is hoped to test its effect on mutation frequency of other unstable pal alleles (Fincham and Harrison, 1967) and on unstable alleles at other loci.

\section{Summary}

1. A gene designated Stabiliser ( $S t$ ) has been identified controlling the mutation frequency of pallida-recurrens ( $p a l^{r e c}$ ) in Antirrhinum majus. St and pal are not linked.

2. St is semi-dominant; the mutation frequency of pal rec is often about 20 times greater with $S t / s t$ than with $S t / S t$ and about 20 times greater again with $s t / s t$, though these ratios are subject to variation.

3. Sectors in $S t / s t$ plants showing the mutability characteristic of $s t / s t$ are sometimes found, suggesting that $S t$ may itself be mutable.

\section{REFERENCES}

FINCHAM, J. R. S., AND HARRISON, B. J. 1967. Instability at the pal locus Antirrhinum majus.

2. Multiple alleles produced by mutation of one original unstable allele. Heredity. $22,211$.

HARRISON, B. J., AND FINCHAM, J. R. s. 1964. Instability at the pal locus in Antirrhinum majus. 1. Effects of environment on frequencies of somatic and germinal mutation. Heredity, $19,237$.

MCCLINTOCK, B. 1965. The control of gene action in maize. Brookhaven Symposia in Biology, $18,162$.

RHOADES, M. M. 1941. The genetic control of mutability in maize. Cold Spring Harbor Symp. Quant. Biol., 9, 138.

Note Added in Proof. In the previous paper in this series (Fincham and Harrison, 1967), Jorgensen and Geissman (Arch. Biochem. Biophys., 54, 72, 1955). were wrongly quoted as having identified quercetin-3-glucoside in ivory flowered plants (homozygous for incolorata). In fact, these authors found this pigment only in magenta flowers and not in ivory flowers. We have observed a component with chromatographic properties similar to quercetin-3-glucoside in a number of inc/inc plants but have not carried out a full identification. The question of the position of the biosynthetic block due to inc must remain open pending further investigation. 


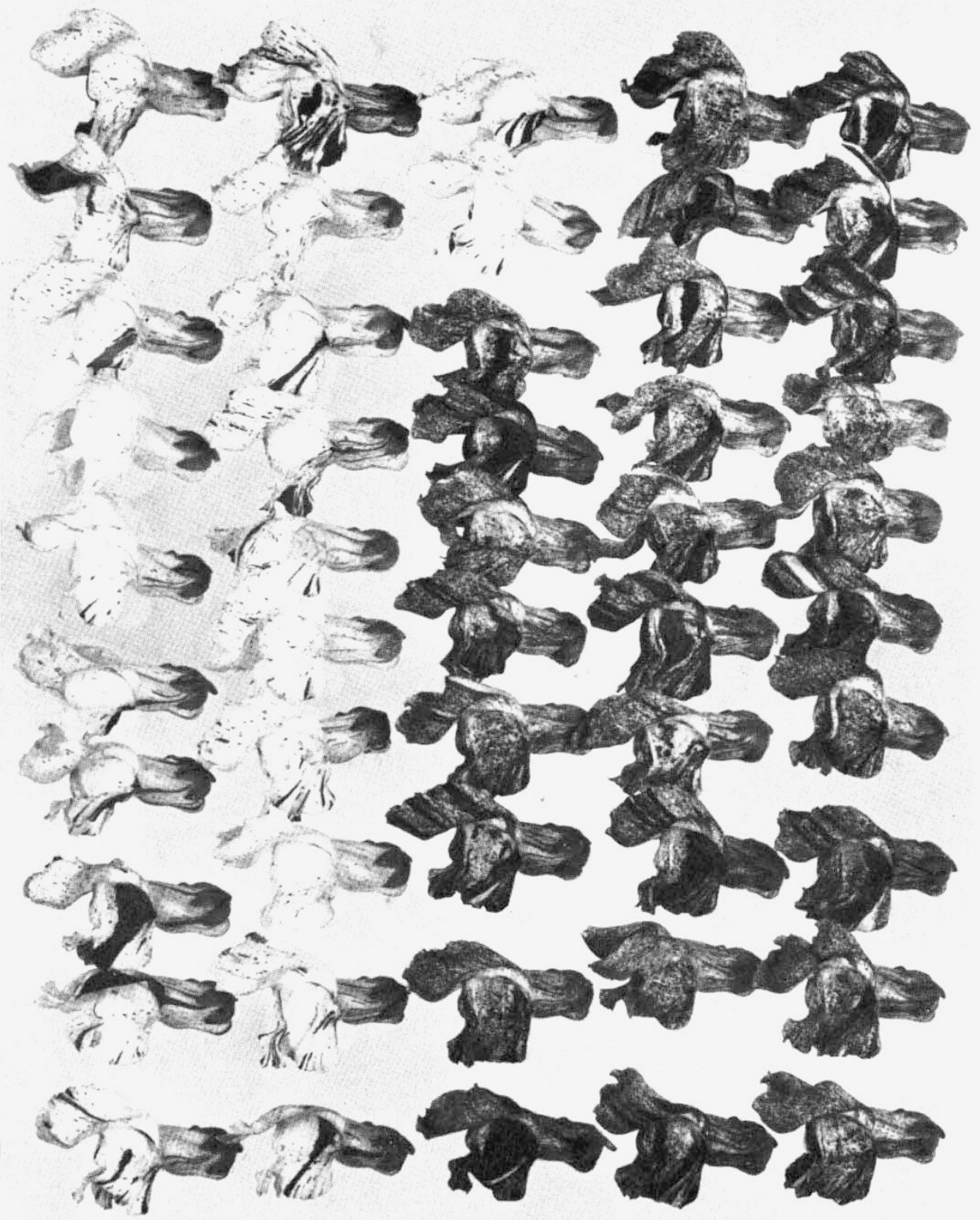

Representative flowers from 28 high- and 22 medium-spotted plants from family T181 (cf. table 2). 
Plate II
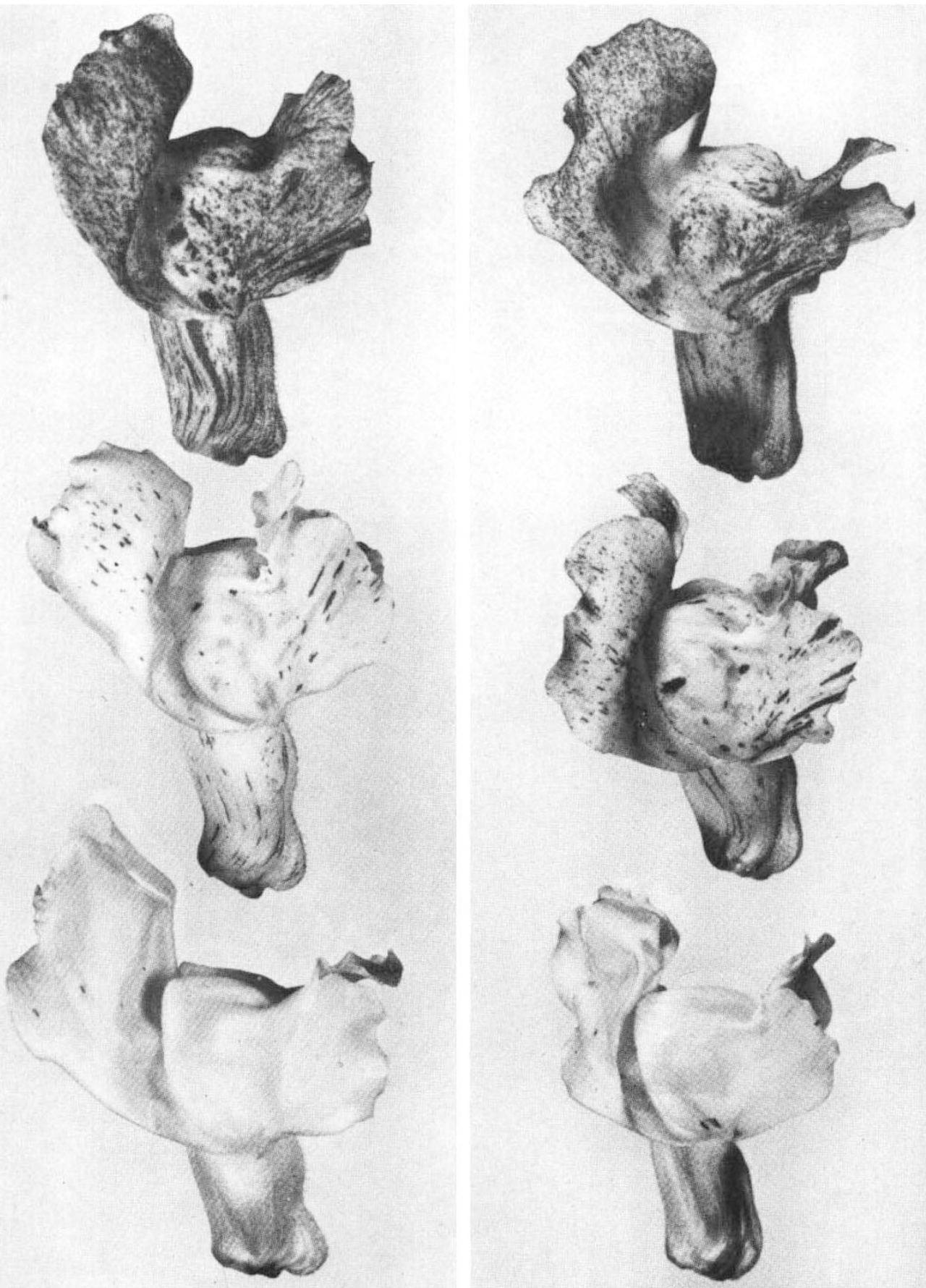

Flowers from st/st, St/st and $S t / S t$ plants (in order top to bottom) all of constitution palrec/paltub. In the left-hand photograph the pal ${ }^{r e c}$ allele was derived originally from the (high) palrec stock and in the right-hand photograph it was from the (low) palrec stock. The families from which the flowers were taken were (top to bottom, left) W64, W66 and W66 and (top to bottom, right) W73, W79 and W69 (of table 4). Spot counts on these six flowers were (in the same order) 16,610,4100, 5, 120 and 4300 . 


\section{Plate III}

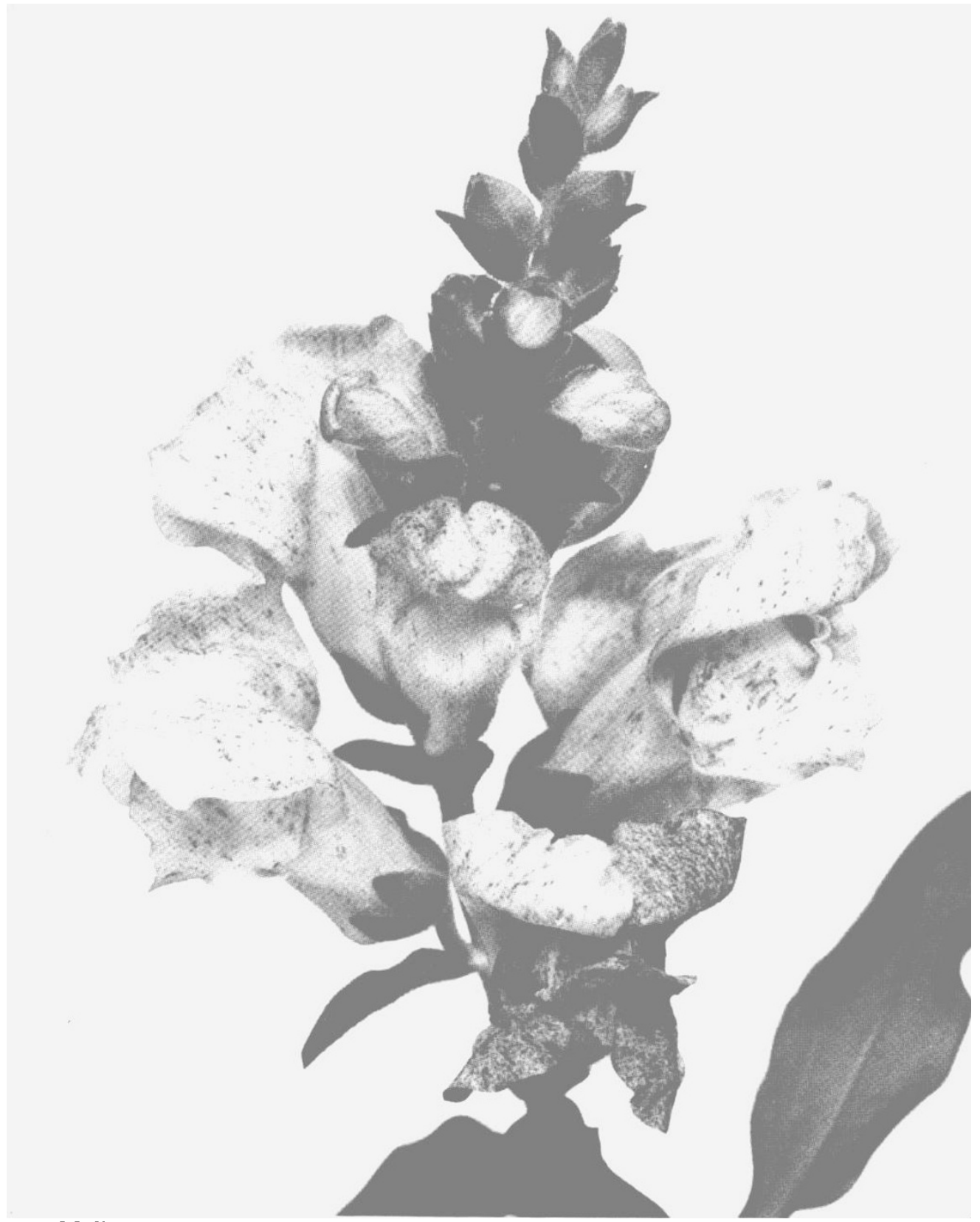

Medium-spotted $(S t / s t)$ plant from family W78 showing a high-spotted (?st/st) sector on the lowest flower. 